\title{
Surface hydrophobicity: effect of alkyl chain length and network homogeneity
}

\author{
Wenqian Chen $(\bowtie)^{1}$, Vikram Karde ${ }^{1}$, Thomas N. H. Cheng' ${ }^{1}$, Siti S. Ramli ${ }^{2}$, Jerry Y. Y. Heng ${ }^{1}$ \\ 1 Department of Chemical Engineering, Imperial College London, London, SW7 2AZ, UK \\ 2 Department of Food Technology, Faculty of Applied Sciences, Universiti Teknologi MARA (UiTM), Shah Alam, Selangor, Malaysia
}

(C) The Author(s) 2020. This article is published with open access at link.springer.com and journal.hep.com.cn

\begin{abstract}
Understanding the nature of hydrophobicity has fundamental importance in environmental applications. Using spherical silica nanoparticles (diameter $=369 \pm 7$ $\mathrm{nm})$ as the model material, the current study investigates the relationship between the alkyl chain network and hydrophobicity. Two alkyl silanes with different chain length (triethoxymethylsilane (C1) vs. trimethoxy(octyl)silane (C8)) were utilised separately for the functionalisation of the nanoparticles. Water contact angle and inverse gas chromatography results show that the alkyl chain length is essential for controlling hydrophobicity, as the octylfunctionalised nanoparticles were highly hydrophobic ( water contact angle $=150.6^{\circ} \pm 6.6^{\circ}$ ), whereas the methylfunctionalised nanoparticles were hydrophilic (i.e., water contact angle $=0^{\circ}$, similar to the pristine nanoparticles). The homogeneity of the octyl-chain network also has a significant effect on hydrophobicity, as the water contact angle was reduced significantly from $148.4^{\circ} \pm 3.5^{\circ}$ to $30.5^{\circ} \pm 1.0^{\circ}$ with a methyl-/octyl-silane mixture (ratio $=$ 160:40 $\mu \mathrm{L} \cdot \mathrm{g}^{-1}$ nanoparticles).
\end{abstract}

Keywords hydrophobicity, surface energy, wettability, alkyl chain network, silica nanoparticle

\section{Introduction}

Due to their low cost, exceptional stability as well as controllable physiochemical properties, silica-based nanostructures have a wide range of environmental applications including oil recovery, carbon dioxide storage and environmental remediation [1-4]. Spherical nanoparticle prepared via the Stöber method is one of the most popular nanostructures due to its high monodispersity in size and shape as well as its versatility for chemical modification [5-13]. The diameter of Stöber nanoparticle

Received February 14, 2020; accepted March 24, 2020

E-mail: wenqian.chen06@imperial.ac.uk can cover the entire nanometre range (i.e., $10 \mathrm{~nm}-1 \mu \mathrm{m}$ ) by controlling the synthesis conditions such as $\mathrm{pH}$ and temperature $[5,6]$. Through methods such as co-condensation and grafting, different chemical groups can be attached to the surface of Stöber nanoparticles [14]. For instance, superhydrophobic Stöber nanoparticles can be obtained by attaching fluorinated copolymers onto their surface [15].

The surface chemistry of silica nanoparticles is of fundamental importance for colloidal stability as it affects the interaction among the nanoparticles themselves as well as between the nanoparticles and the surrounding [16]. In the case of Pickering emulsions, it was found that nanoparticles with an intermediate degree of hydrophobicity (i.e., a water contact angle $\sim 90^{\circ}$ ) could stabilise droplets in water-in-oil and oil-in-water systems $[17,18]$. Typically, the intermediate degree of hydrophobicity is achieved by grafting nanoparticles with both hydrophobic and hydrophilic silanes or a mixed polymer silane with similar nature [19-24].

In this study, we demonstrate the nature of hydrophobicity as the result of an interacting network among the hydrophobic chains on the surface of Stöber nanoparticles. Instead of using both hydrophobic and hydrophilic silanes as in the existing literature, two similar silanes with different alkyl chain lengths (i.e., C1 vs. C8) were mixed in various proportions and grafted onto Stöber nanoparticles. Various techniques including contact angle measurement, inverse gas chromatography (IGC), thermal gravimetric analysis and helium pycnometry were used to characterise the nanoparticles in order to analyse the effect of chemical modification.

\section{Experimental}

\subsection{Materials}

All chemicals were used without purification unless stated 
otherwise. Tetraethyl orthosilicate (TEOS) (98\%), trimethoxy(octyl)silane (96\%), triethoxymethylsilane (99\%) were purchased from Sigma Aldrich (Gillingham, UK). Ethanol $(\geqslant 99.8 \%)$, acetone $(\geqslant 99.8 \%)$ and sodium hydroxide were purchased from VWR (Lutterworth, UK). Cyclohexane $(\geqslant 99.8 \%)$ was purchased from Fisher Chemical (Loughborough, UK). De-ionised water was supplied by PURELAB Chorus 1 (ELGA LabWater) water purification system. More details can be found in Table S1 (cf. Electronic Supplementary Material, ESM).

For the characterisation of silica nanoparticles, carboncoated copper transmission electron microscopy (TEM) grid was supplied by EM Resolutions (Sheffield, UK) and carbon conductive adhesive tape was supplied by Agar Scientific (Stansted, UK). Silanised glass wool was purchased from Sigma-Aldrich (Gillingham, UK).

\subsection{Synthesis of silica nanoparticle}

Silica nanoparticles were synthesised with the Stöber method $[5,6]$. TEOS $(60 \mathrm{~mL})$ was added into ethanol/deionised water mixture $(960 \mathrm{~mL} / 138 \mathrm{~mL})$ at $40{ }^{\circ} \mathrm{C}$, followed by $0.1 \mathrm{~mol} \cdot \mathrm{L}^{-1} \mathrm{NaOH}(42 \mathrm{~mL})$. The final mixture was stirred overnight at $40{ }^{\circ} \mathrm{C}$. The mixture was centrifuged $\left(6000 \mathrm{r} \cdot \mathrm{min}^{-1}, 10 \mathrm{~min}\right)$ to recover the synthesised silica nanoparticles, which were dried at room temperature prior to further modification.

\subsection{Modification of silica nanoparticle}

The dried silica nanoparticles $(1.0 \mathrm{~g})$ were added into cyclohexane $(100 \mathrm{~mL})$, followed by the target silanes (i.e., trimethoxy(octyl)silane and triethoxymethylsilane) (5$200 \mu \mathrm{L}$ ). The mixture was sonicated at room temperature for $10 \mathrm{~min}$ and refluxed overnight (boiling point of cyclohexane $\left.=81{ }^{\circ} \mathrm{C}\right)$. The mixture was cooled to room temperature and centrifuged $\left(6000 \mathrm{r} \cdot \mathrm{min}^{-1}, 10 \mathrm{~min}\right)$ to recover the modified silica nanoparticles. The recovered nanoparticles were washed repeatedly with fresh acetone and dried at room temperature and finally in vacuum oven $\left(60{ }^{\circ} \mathrm{C}, 100\right.$ mbar) overnight.

\subsection{Characterisation of silica nanoparticle}

The shape and size of silica nanoparticles were confirmed with TEM and scanning electron microscopy (SEM). For TEM analysis, silica nanoparticles were sonicated in ethanol for proper dispersion. One drop of the sonicated mixture was placed onto a carbon-coated copper TEM grid to be dried naturally. The on-grid sample was analysed with a 2100 Plus transmission electron microscope (JEOL USA, Inc., USA). For SEM analysis, silica nanoparticles were spread on the carbon conductive adhesive tape and coated with $15 \mathrm{~nm}$ chromium in a Q150T turbomolecularpumped coating system (Quorum Technologies Ltd., UK) (sputter current $=120 \mathrm{~mA}$, tooling factor $=2.43$ and maximum sputtering time $=4 \mathrm{~min}$ ). The chromium-coated sample was analysed with a Leo Gemini 1525 scanning electron microscope (Carl Zeiss AG, Germany) with an accelerating voltage of $5 \mathrm{kV}$.

The skeletal density of silica nanoparticles was measured at $25{ }^{\circ} \mathrm{C}$ by helium pycnometry with an AccuPyc II 1340 pycnometer (Micromeritics Instrument Corp., USA).

The hydrophobicity of silica nanoparticles was quantified by sessile drop contact angle and IGC measurements. For sessile drop contact angle measurement, silica nanoparticles were further dried in a vacuum oven $\left(60{ }^{\circ} \mathrm{C}, 100 \mathrm{mbar}\right.$ ) overnight and compressed into tablets using a R500 system (Gamlen Tableting Ltd., UK) $(100 \mathrm{mg}$ sample, target $\mathrm{load}=200 \mathrm{~kg}$, speed $=$ $\left.8 \mathrm{~mm} \cdot \mathrm{min}^{-1}\right)$. Contact angle was measured with a Model 590 goniometer/tensiometer (Ramé-hart Instrument Co., USA) at room temperature using de-ionised water as the test liquid. One drop of water $(10 \mu \mathrm{L})$ was placed on the tablet and the static contact angle was measured over 100 seconds with 1 second measurement interval.

All the IGC measurements were conducted at infinite dilution with an IGC surface energy analyser (Surface Measurement Systems, UK). The sample $(\sim 50 \mathrm{mg})$ was packed into a silanised glass column (length $=300 \mathrm{~mm}$, internal diameter $=3 \mathrm{~mm}$ ), which was plugged with silanised glass wool on both ends. A jolting voltameter (Surface Measurement Systems, UK) was used to provide mechanical tapping to the sample in order to ensure good powder packing in the column. The packed sample column was placed into the column oven. The carrier gas was helium at a flow rate of $10 \mathrm{~mL} \cdot \mathrm{min}^{-1}$ and the reference gas was methane to determine the dead volume. Before each measurement, the sample column was conditioned at $30^{\circ} \mathrm{C}$ and $0 \%$ relative humidity for $2 \mathrm{~h}$ at $10 \mathrm{~mL} \cdot \mathrm{min}^{-1}$ carrier gas flow rate. All the measurements were performed at $30{ }^{\circ} \mathrm{C}$ and $0 \%$ relative humidity (i.e., same as the analysis conditions). Polar probes including ethyl acetate and dichloromethane, and $n$-alkane probes including decane, nonane, octane, heptane and hexane were used under infinite dilution of $3 \%$ probe coverage.

The retention behaviour of the probes was used to calculate the dispersive component $\left(\gamma_{\mathrm{d}}\right)$ of surface energy with the Schultz approach, whereas the acid-base $\left(\gamma_{\mathrm{ab}}\right)$ component of surface energy was determined using Della Volpe approach $[25,26]$. Firstly, $V_{\mathrm{N}}$ was determined with Eq. (1). The free energy of adsorption $\left(\Delta G_{\mathrm{a}}\right)$ was determined from $V_{\mathrm{N}}$ with Eq. (2). The dispersive surface free energy $\left(\gamma_{\mathrm{s}}^{\mathrm{d}}\right)$ is related to $V_{\mathrm{N}}$ of the non-polar alkane solute according to Eq. (3) [25]. To determine $\gamma_{\mathrm{s}}{ }^{\mathrm{d}}$, a linear plot of $\ln V_{\mathrm{N}}$ against $\left(\gamma_{1}^{\mathrm{d}}\right)^{0.5}$ of the $n$-alkane probes was made and the slope of the best fit line was used to calculate $\gamma_{\mathrm{s}}^{\mathrm{d}}$.

$$
V_{\mathrm{N}}=F \frac{J}{m} t_{\mathrm{R}} \frac{T}{273.15}
$$


where $F$ is the carrier gas flow rate, $J$ is the James and Martin pressure correction factor for gas compressibility, $m$ is the mass of the sample, $t_{\mathrm{R}}$ is the retention time of the probe, $T$ is the column temperature.

$$
\Delta G_{\mathrm{a}}=-\mathrm{R} T \ln V_{\mathrm{N}}+\mathrm{K},
$$

where $\mathrm{R}$ is gas constant and $\mathrm{K}$ is a constant.

$$
-\Delta G_{\mathrm{a}}^{\mathrm{d}}=\mathrm{R} T \ln V_{\mathrm{N}}=2 N a\left(\gamma_{1}^{\mathrm{d}}\right)^{0.5}\left(\gamma_{\mathrm{s}}^{\mathrm{d}}\right)^{0.5}+\mathrm{K}^{\prime},
$$

where $N$ is Avogadro's number, $a$ is the cross-sectional area of solute, $\gamma_{1}^{\mathrm{d}}$ is the Lifshitz-van der Waals dispersive surface free energy of the probe and $\mathrm{K}^{\prime}$ is another constant that is different from the constant $\mathrm{K}$ in Eq. (2).

To determine the acid-base surface free energy $\left(\gamma_{\mathrm{s}}^{\mathrm{ab}}\right)$, it was necessary to determine the acid-base component of the free energy of adsorption for polar solutes $\left(\Delta G^{\mathrm{ab}}\right)$ from the difference between the alkane regression line and the polar probe according to Eq. (4). According to Das et al. [27], $\Delta G^{\mathrm{ab}}$ of monopolar acid and monopolar base probes were used to calculate the acidic and basic parameters of the solid surface energy $\left(\gamma_{\mathrm{s}}^{+}\right.$and $\gamma_{\mathrm{s}}^{-}$respectively based on Della Volpe scale) based on the Van Oss-Good theory [28] with Eq. (5). $\gamma_{\mathrm{s}}^{\mathrm{ab}}$ was determined with Eq. (6). The total surface energy $\left(\gamma_{\mathrm{s}}{ }^{\text {total }}\right)$ was the sum of $\gamma_{\mathrm{s}}{ }^{\mathrm{d}}$ and $\gamma_{\mathrm{s}}{ }^{\text {ab }}$ (Eq. (7)).

$$
\begin{gathered}
\Delta G_{\mathrm{a}}=\Delta G_{\mathrm{a}}^{\mathrm{d}}+\Delta G_{\mathrm{a}}^{\mathrm{ab}}, \\
\Delta G_{\mathrm{a}}^{\mathrm{ab}}=2 N a\left(\left(\gamma_{1}^{+} \gamma_{\mathrm{s}}^{-}\right)^{0.5}+\left(\gamma_{1}^{-} \gamma_{\mathrm{s}}^{+}\right)^{0.5}\right), \\
\gamma_{\mathrm{s}}^{\mathrm{ab}}=2\left(\gamma_{\mathrm{s}}^{+} \gamma_{\mathrm{s}}^{-}\right)^{0.5}, \\
\gamma_{\mathrm{s}}^{\text {total }}=\gamma_{\mathrm{s}}{ }^{\mathrm{d}}+\gamma_{\mathrm{s}}^{\mathrm{ab}} .
\end{gathered}
$$

\section{Results and discussion}

\subsection{Surface hydrophobicity: effect of alkyl chain length}

TEM analysis showed that the synthesised Stöber nanoparticles had an average diameter of $369 \pm 7 \mathrm{~nm}$ and a smooth surface (Fig. 1(a)), whereas SEM analysis (Fig. 1(b)) confirmed the high monodispersity of size and shape as expected from the highly robust Stöber method [5]. The skeletal density of the pristine Stöber nanoparticles measured by helium pycnometry was $2.03 \mathrm{~g} \cdot \mathrm{cm}^{-3}$, which was consistent with the value reported in literature [16].

Functionalising the nanoparticles with triethoxymethylsilane and trimethoxy(octyl)silane (Fig. 2) changed the hydrophobicity of nanoparticles to various extent. As shown in Fig. 3(a), the pristine nanoparticles had a $0^{\circ}$ contact angle as the water droplet was instantly absorbed by the tablet prepared from the pristine nanoparticles. Similar results were obtained for the methyl-functionalised nanoparticles regardless of the quantity of triethoxymethylsilane per gram of nanoparticles during functionalisation. In contrast, the water contact angle of octylfunctionalised nanoparticles remained close to the superhydrophobic level (between $144.1^{\circ} \pm 0.4^{\circ}$ and $\left.158.1^{\circ} \pm 0.2^{\circ}\right)$ as the silane to nanoparticle ratio during functionalisation increased from 5 to $200 \mu \mathrm{L} \cdot \mathrm{g}^{-1}$. This demonstrates the effectiveness of the trimethoxy(octyl) silane in forming a hydrophobic coating on the hydrophilic silica nanoparticle.

With IGC, the surface energy of pristine and functionalised silica nanoparticles can be determined with higher accuracy and sensitivity than the contact angle method to provide a fundamental understanding about the effect of chemical modification on the wettability of the nanoparticle [29-33]. The calculated surface energy for pristine, methyl- and octyl-functionalised silica nanoparticles is presented in Fig. 4.

Firstly, the chemical modification with silanes resulted in an observable reduction (i.e., maximum $8 \%$ ) in the total surface energy from $53.2 \mathrm{~mJ} \cdot \mathrm{m}^{-2}$ for the pristine nanoparticle to $48.7-49.6 \mathrm{~mJ} \cdot \mathrm{m}^{-2}$ for the methyl-functionalised nanoparticle, and to $49.0-51.5 \mathrm{~mJ} \cdot \mathrm{m}^{-2}$ for the octyl-functionalised nanoparticles. This provides evidence for the effectiveness of chemical modification, which is especially important for the methyl-functionalised nanoparticle, as water contact angle measurement is unable to distinguish it from the pristine nanoparticle. By separating the total surface energy into the dispersive and acid-base
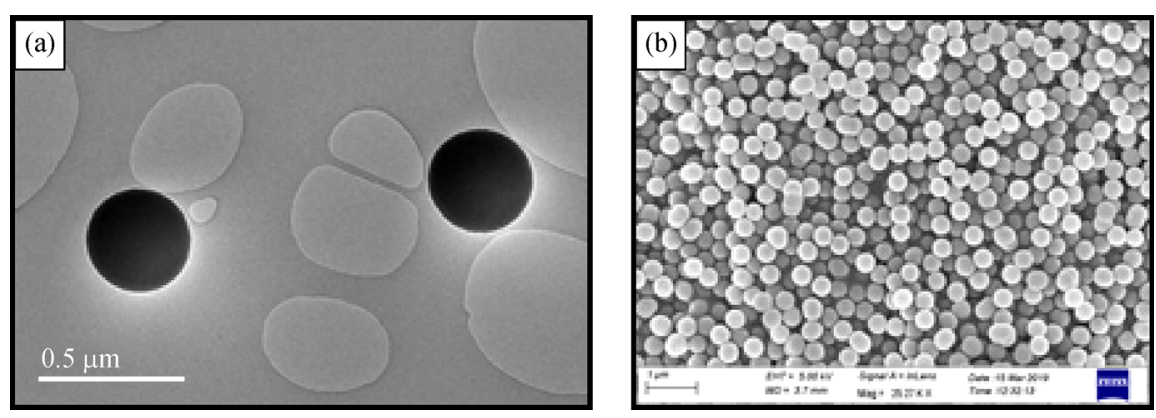

Fig. 1 (a) TEM and (b) SEM images of Stöber nanoparticles. 

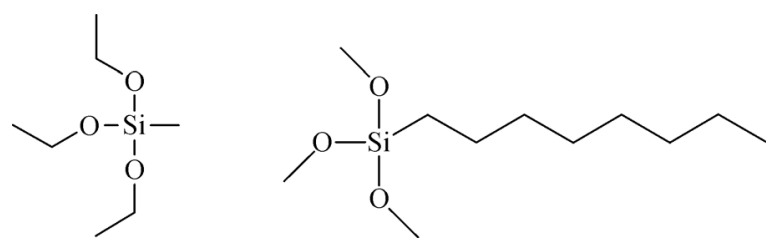

Fig. 2 Triethoxymethylsilane (left) and trimethoxy(octyl)silane (right).

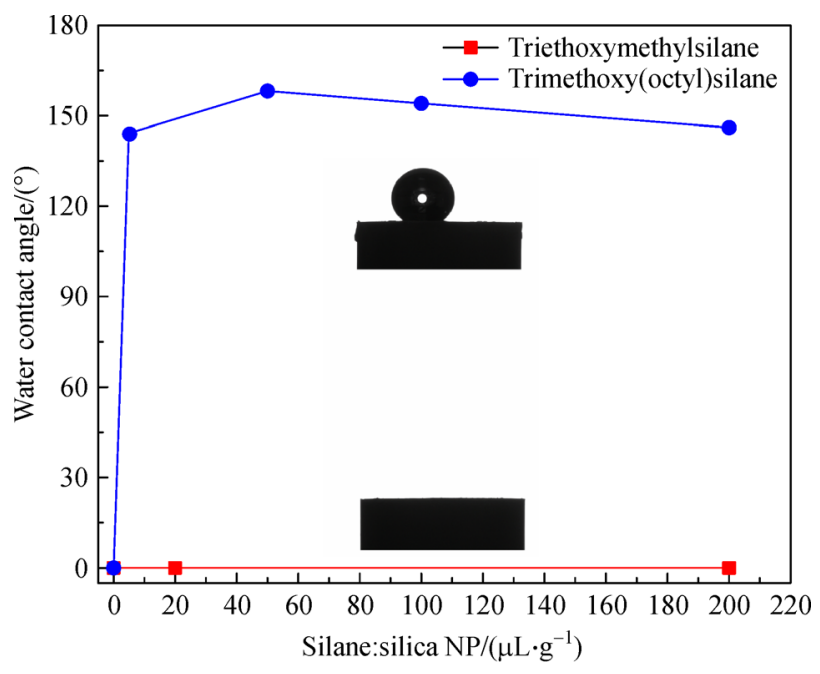

Fig. 3 Water contact angle for pristine (i.e., silane:silica $\mathrm{NP}=$ $0 \mu \mathrm{L} \cdot \mathrm{g}^{-1}$ ) and modified nanoparticles (pictures showing water droplet on tablet or just tablet after the absorption of water droplet). Note: error bars are too small to be visible (e.g., $158.1^{\circ} \pm 0.2^{\circ}$ ).

components (i.e., $\gamma_{\mathrm{s}}^{\mathrm{d}}$ and $\gamma_{\mathrm{s}}^{\mathrm{ab}}$ ), it is obvious that reduction of total surface energy was mainly attributed to the reduction in the dispersive surface energy, which was $43.3 \mathrm{~mJ} \cdot \mathrm{m}^{-2}$ for the pristine nanoparticle and $39.1-42.5 \mathrm{~mJ} \cdot \mathrm{m}^{-2}$ for the functionalised nanoparticle. Similar observation was previously made for the functionalisation of pharmaceutical excipient powder, where the reduction in the dispersive surface energy $\left(\gamma_{\mathrm{d}}\right)$ was attributed to the passivation of high energy sites by the covalently attached silanes [31].

Secondly, while the total surface energy of methyl- and octyl-functionalised nanoparticles was in the similar range (approximately $49 \mathrm{~mJ} \cdot \mathrm{m}^{-2}$ ), there was observable difference in the acid-base surface energy $\left(\gamma_{\mathrm{s}}^{\mathrm{ab}}\right)$ of the functionalised nanoparticles, which resulted in the significant difference in water contact angle (Fig. 3). For the methyl-functionalised nanoparticle with different triethoxymethylsilane to nanoparticle ratios, the acid-base surface energy $\left(\gamma_{\mathrm{s}}^{\mathrm{ab}}\right)$ was $9.1-9.6 \mathrm{~mJ} \cdot \mathrm{m}^{-2}$, slightly lower than the value of the pristine nanoparticle $\left(9.9 \mathrm{~mJ} \cdot \mathrm{m}^{-2}\right)$. As the ratio of trimethoxy(octyl)silane to nanoparticle increased from 5 to $200 \mu \mathrm{L} \cdot \mathrm{g}^{-1}$, the acid-base surface energy $\left(\gamma_{\mathrm{ab}}\right)$ of the resulting octyl-functionalised nanoparticle decreased significantly from 9.0 to $7.8 \mathrm{~mJ} \cdot \mathrm{m}^{-2}$, even though the water contact angle remained relatively constant (between
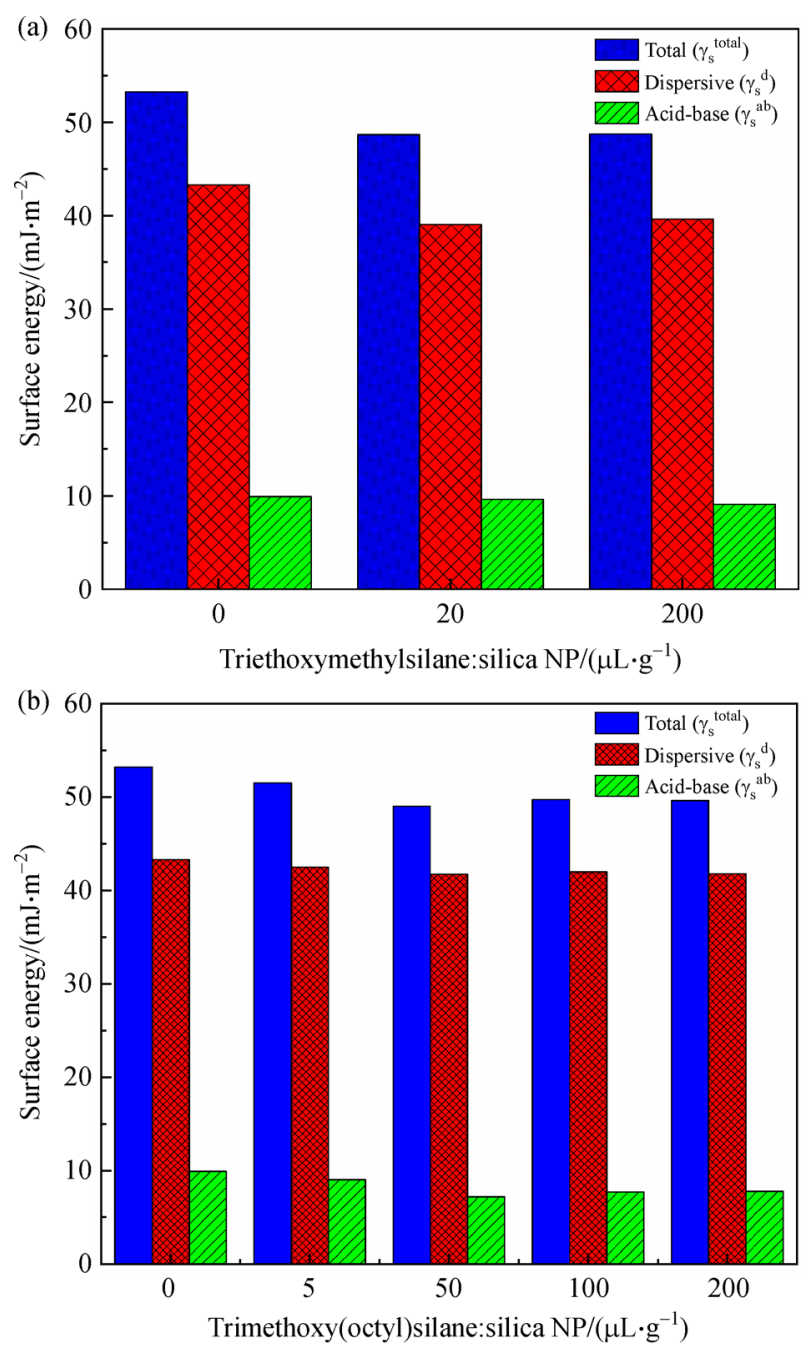

Fig. 4 Surface energy of pristine (i.e., silane:silica NP=0), (a) methyl- and (b) octyl-functionalised silica nanoparticles.

$144.1^{\circ} \pm 0.4^{\circ}$ and $\left.158.1^{\circ} \pm 0.2^{\circ}\right)$. The high water contact angle was probably due to the hydrophobic network formation by the octyl-chains as discussed in more detail below, showing the effectiveness of this hydrophobic network in shielding the nanoparticle surface from water.

As the particle diameter $(369 \pm 7 \mathrm{~nm})$ is significantly larger than the length of the alkyl chains, the surface of nanoparticle can be treated as a flat surface with respect to the alkyl chains [21]. The hydrophilicity of pristine nanoparticles originates from the free silanol groups 
$(-\mathrm{SiOH})$ available on the surface as well as within the nanoparticles (Fig. 5(a)). As the water droplet was placed onto the tablet of pristine nanoparticles, the water readily wetted the surface of the nanoparticles and occupied the interparticle voids, resulting in the instant absorption or imbibition of water droplet.

In contrast, due to the length of the octyl chain $\left(-\left(\mathrm{CH}_{2}\right)_{7}-\mathrm{CH}_{3}\right)$ (Fig. 5(c)), it is easier to form a hydrophobic network for shielding the nanoparticle from water, resulting in a high water contact angle. The thermal gravimetric analysis results of the pristine and functionalised nanoparticles (silane:silica ratio $=200 \mu \mathrm{L} \cdot \mathrm{g}^{-1}$ for both silanes) show a similar trend for mass loss over a wide range of temperature $\left(100^{\circ} \mathrm{C}\right.$ to $\left.800^{\circ} \mathrm{C}\right)$, which agree with previous studies on the thermal properties of pristine and functionalised silica nanoparticles [34-37]. The similar mass loss for both pristine and functionalised nanoparticles indicate that the mass of the methyl- and octyl-functional groups is negligible with respect to the mass of the nanoparticle (Fig. 6). In other words, even though the octyl-network is only a very thin layer on the surface of the nanoparticle, it is highly effective in preventing the contact of water with the nanoparticle. Unlike the octyl chain, the methyl chain $\left(-\mathrm{CH}_{3}\right)$ is much shorter and hence is unable to form a hydrophobic network despite the increase in

(a)

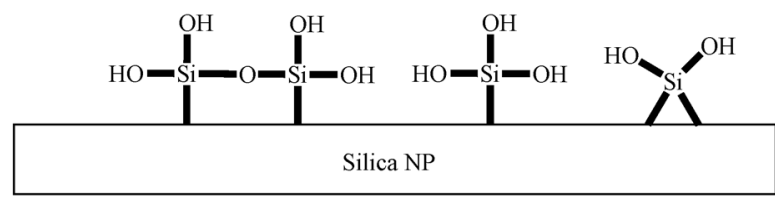

(b)

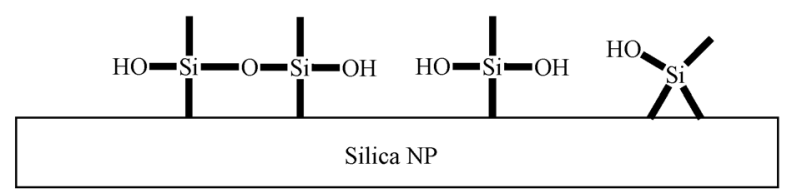

(c)

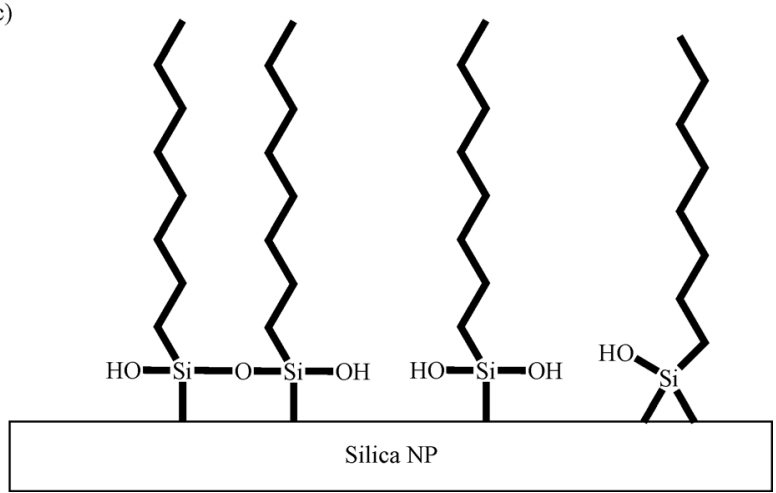

Fig. 5 The surface of (a) pristine, (b) methyl-functionalised and (c) octyl-functionalised silica nanoparticles.

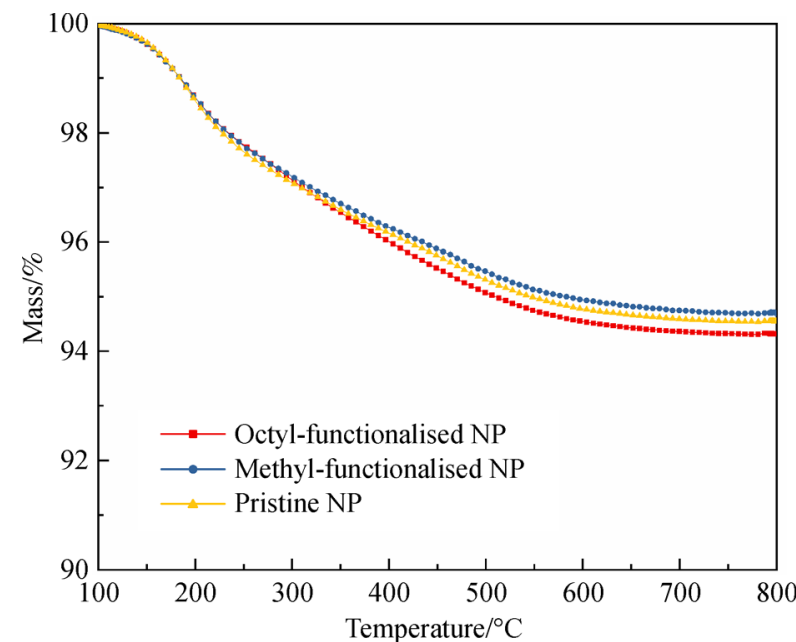

Fig. 6 Thermal gravimetric analysis results for pristine and modified nanoparticles (silane:silica nanoparticle ratio $=$ $200 \mu \mathrm{L} \cdot \mathrm{g}^{-1}$ for both silanes).

coverage density achieved with the increase in the silane to silica nanoparticle ratio during functionalisation (Fig. 7). As a result, water still readily wetted the methylfunctionalised nanoparticles due to the accessibility of the free silanol groups of the methyl-silane and nanoparticle surface (Fig. 5(b)).

3.2 Surface hydrophobicity: effect of alkyl chain network homogeneity

Besides the length of alkyl chain, the homogeneity of the functional group has an important effect on the surface hydrophobicity of the nanoparticle. Figure 8(a) shows the water contact angles of octyl-functionalised nanoparticles prepared with pure trimethoxy(octyl)silane and a mixture of triethoxymethylsilane and trimethoxy(octyl)silane (in the latter case, the total silane to nanoparticle ratio was kept constant at $200 \mu \mathrm{L} \cdot \mathrm{g}^{-1}$ (e.g., $100 \mu \mathrm{L} \cdot \mathrm{g}^{-1}$ trimethoxy(octyl) silane $+100 \mu \mathrm{L} \cdot \mathrm{g}^{-1}$ triethoxymethylsilane)). The octylfunctionalised nanoparticles prepared with the pure trimethoxy(octyl)silane remained highly hydrophobic (i.e., water contact angle $>90^{\circ}$ ) even with the silane to nanoparticle ratio as low as $5 \mu \mathrm{L} \cdot \mathrm{g}^{-1}$ (Figs. S1-S8, cf. ESM). This is in stark contrast with the nanoparticles functionalised with the silane mixture, whose water contact angle decreased drastically from $154.1^{\circ} \pm 0.3^{\circ}$ to $30.5^{\circ} \pm 1.0^{\circ}$ as the trimethoxy(octyl)silane to nanoparticle ratio was reduced to $40 \mu \mathrm{L} \cdot \mathrm{g}^{-1}$.

The surface energy of silica nanoparticles functionalised by mixtures of triethoxymethylsilane and trimethoxy (octyl)silane is shown in Fig. 9. Similar to the nanoparticles functionalised by pure trimethoxy(octyl)silane (Fig. 4(b)), the dispersive surface energy remained relatively constant regardless of the ratio of triethoxymethylsilane and trimethoxy(octyl)silane, whereas the 


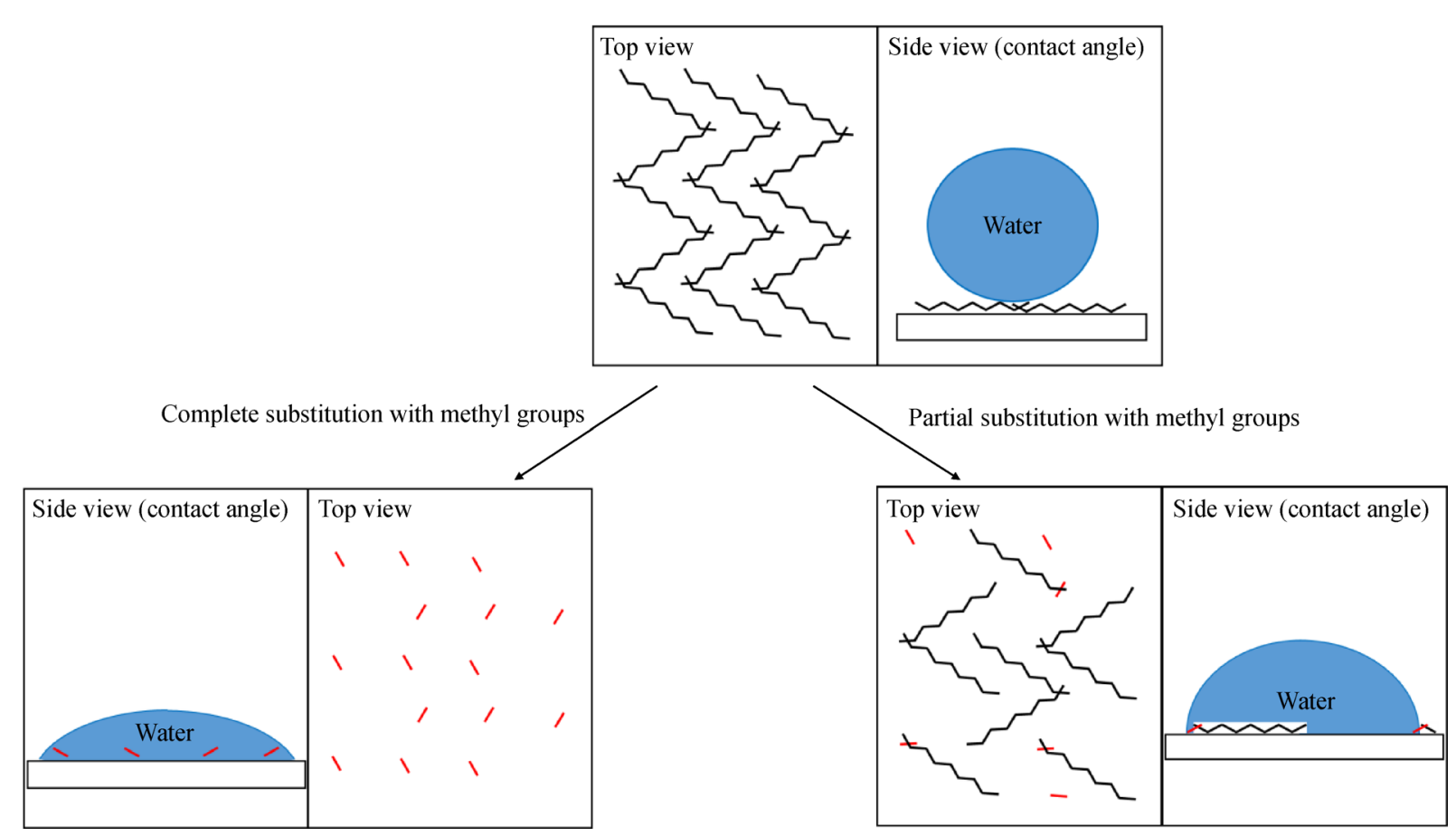

Fig. 7 Surface of nanoparticle functionalised with the same coverage density of octyl- and methyl-functional groups (i.e., before and after complete substitution with methyl groups).

acid-base surface energy $\left(\gamma_{\mathrm{s}}^{\mathrm{ab}}\right)$ had a general decreasing trend $\left(9.1 \mathrm{~mJ} \cdot \mathrm{m}^{-2}\right.$ to $\left.7.9 \pm 0.3 \mathrm{~mJ} \cdot \mathrm{m}^{-2}\right)$ as the ratio of trimethoxy(octyl)silane to nanoparticle increased (Fig. 8(b)). The decrease in acid-base surface energy $\left(\gamma_{\mathrm{s}}^{\mathrm{ab}}\right)$ corresponded to the extent of functionalisation and the reduction in free silanol group $(-\mathrm{SiOH})$ that could interact favourably with water, leading to a general increasing trending for water contact angle.

The acid-base surface energy $\left(\gamma_{\mathrm{s}}^{\mathrm{ab}}\right)$ data in Fig. 8(b) seem to suggest the presence of an additional factor that influenced the wettability phenomena, as the acid-base surface energy $\left(\gamma_{\mathrm{s}}^{\mathrm{ab}}\right)$ for nanoparticles functionalised with silane mixtures (trimethoxy(octyl)silane:nanoparticle $=$ 40-200 $\mu \mathrm{L} \cdot \mathrm{g}^{-1}$ ) was in a similar range (i.e., $8.2 \mathrm{~mJ} \cdot \mathrm{m}^{-2}$ $\left(40 \mu \mathrm{L} \cdot \mathrm{g}^{-1}\right)$ to $\left.7.9 \pm 0.3 \mathrm{~mJ} \cdot \mathrm{m}^{-2}\left(60-200 \mu \mathrm{L} \cdot \mathrm{g}^{-1}\right)\right)$, but the water contact angle increased significantly from $30.5^{\circ} \pm 1.0^{\circ}\left(40 \mu \mathrm{L} \cdot \mathrm{g}^{-1}\right)$ to $148.4^{\circ} \pm 3.5^{\circ}(60-200 \mu \mathrm{L}$ $\cdot \mathrm{g}^{-1}$ ). This is further confirmed by comparing the water contact angle measurements for nanoparticle functionalised with pure trimethoxy(octyl)silane (silane:nanoparticle $\left.=5 \mu \mathrm{L} \cdot \mathrm{g}^{-1}\right)$ and those functionalised with silane mixture (trimethoxy(octyl)silane:nanoparticle $=40 \mu \mathrm{L}$ $\left.\cdot \mathrm{g}^{-1}\right)$. Although the former had a significantly higher acid-base surface energy $\left(9.0 \mathrm{~mJ} \cdot \mathrm{m}^{-2}\right)$ than the latter $\left(8.2 \mathrm{~mJ} \cdot \mathrm{m}^{-2}\right)$ due to the presence of more free silanol group $(-\mathrm{SiOH})$, its water contact angle $\left(144.1^{\circ} \pm 0.4^{\circ}\right)$ was still significantly higher than the latter $\left(30.5^{\circ} \pm 1.0^{\circ}\right)$.

We propose the homogeneity or extent of defects of the octyl-chain network as the additional factor. Although the chemical functionalisation method used was relatively successful, it was highly unlikely that all the free silanol group $(-\mathrm{SiOH})$ was eliminated. As discussed earlier in this article, the hydrophobicity of nanoparticle surface is the result of the octyl-chain network, whose density determines its effectiveness in shielding the residual silanol group $(-\mathrm{SiOH})$ on the nanoparticle surface from water. Defect occurs when some octyl-chains are replaced with the much shorter methyl-chains, creating hydrophilic patches within this network (Fig. 7-before and after particle substitution with methyl groups). The effect of such defects was negligible for the trimethoxy(octyl)silane to triethoxymethylsilane ratio as low as $60: 140(\mathrm{v} / \mathrm{v})$ (Fig. 8), suggesting the robustness of the hydrophobic octyl-chain network as seen from the high water contact angle $\left(144.1^{\circ} \pm 0.4^{\circ}\right)$ of nanoparticle functionalised with pure trimethoxy(octyl)silane (silane:nanoparticle $=$ $\left.5 \mu \mathrm{L} \cdot \mathrm{g}^{-1}\right)$. However, as the proportion of triethoxymethylsilane continued to increase in the silane mixture, the inhomogeneous or defect-rich octyl-chain network reached a tipping point where the hydrophilic patches could form an interconnected network, resulting in a significant decrease in water contact angle as the triethoxymethylsilane to nanoparticle ratio reached $160 \mu \mathrm{L} \cdot \mathrm{g}^{-1}$.

The results presented in this article are highly relevant for understanding the nature of hydrophobicity and the control strategy. For many of the existing studies that utilised mixtures of hydrophilic and hydrophobic silanes to reduce the hydrophobicity of the target surface, the current findings suggest that the reduction in hydrophobicity was partly due to the disruption of the hydrophobic network 

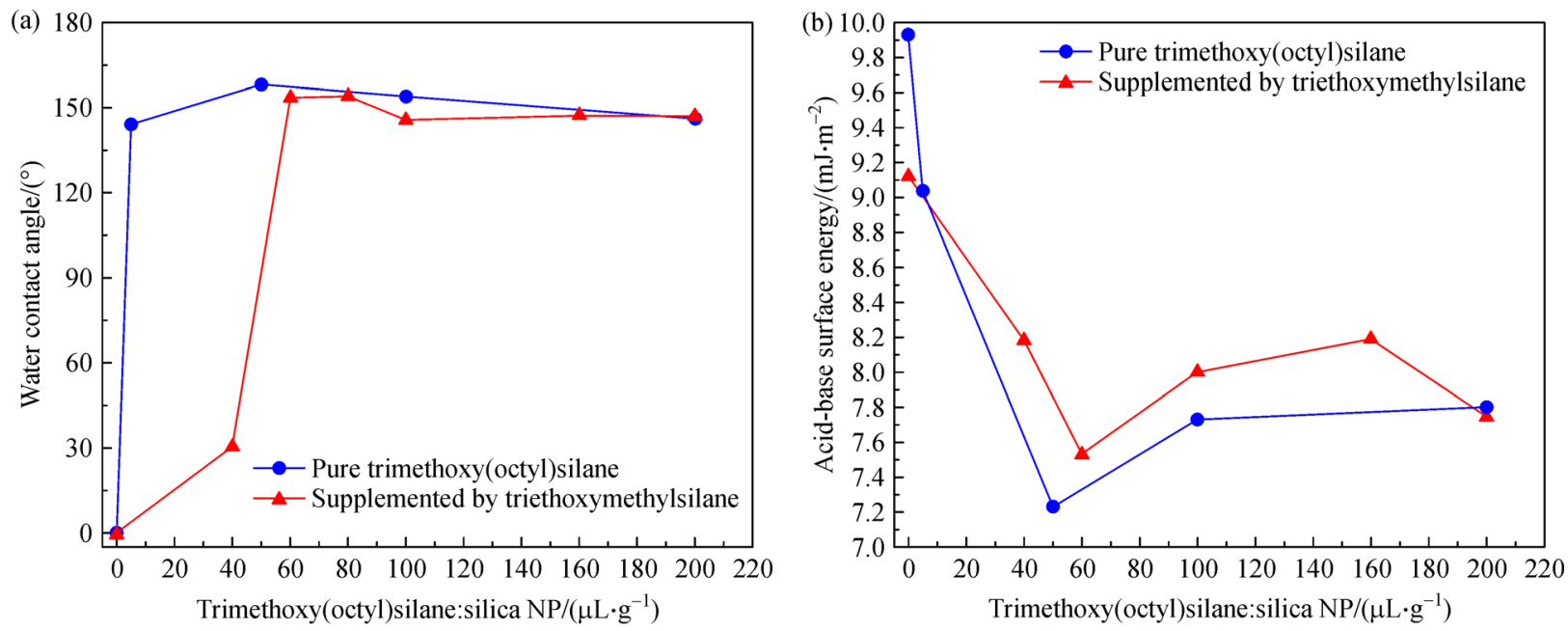

Fig. 8 (a) Water contact angle and (b) acid-base surface energy $\left(\gamma_{\mathrm{AB}}\right)$ for modified nanoparticles (total silane:silica $\mathrm{NP}=200 \mu \mathrm{L} \cdot \mathrm{g}^{-1}$ ). Note: error bars are too small to be visible.

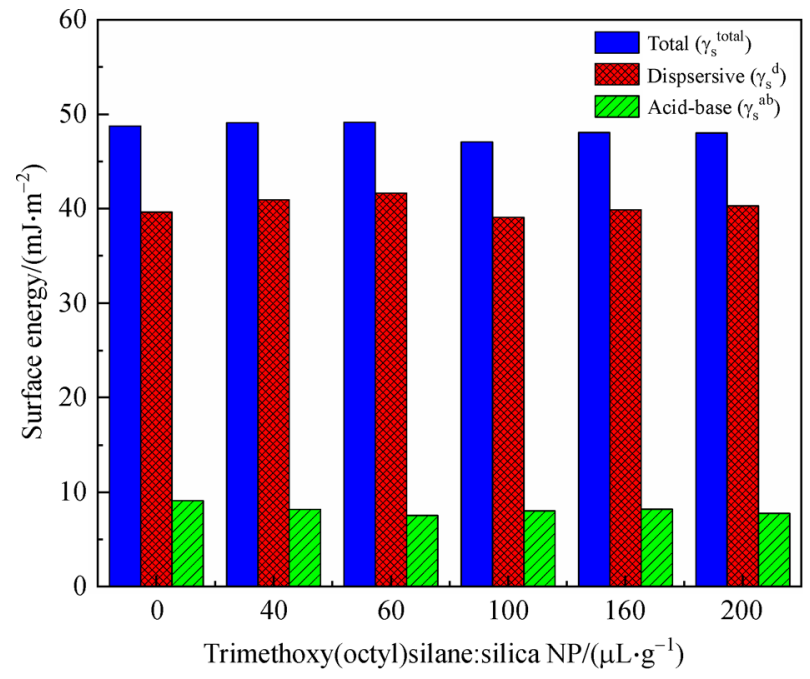

Fig. 9 Surface energy of silica nanoparticles functionalised with triethoxymethylsilane and trimethoxy(octyl)silane mixtures (total silane:silica $\left.\mathrm{NP}=200 \mu \mathrm{L} \cdot \mathrm{g}^{-1}\right)$.

and partly due to the presence of the hydrophilic groups. For studies focusing on the design of superhydrophobic surface, the current findings reveal the important design variables including alkyl chain length and homogeneity of the hydrophobic network.

\section{Conclusions}

In this study, spherical silica nanoparticles were functionalised with silanes that contain methyl- and octylfunctional groups. While the pristine silica nanoparticles were hydrophilic (water contact angle $=0^{\circ}$ ) due to the presence of free silanol groups, the octyl-functionalised nanoparticles were highly hydrophobic (water contact angle between $144.1^{\circ} \pm 0.4^{\circ}$ and $158.1^{\circ} \pm 0.2^{\circ}$ ), demonstrating the effectiveness of the octyl-chain network in shielding the hydrophilic nanoparticle from water. In contrast, methyl-functionalised nanoparticles were hydrophilic (water contact angle $=0^{\circ}$ ) regardless of the triethoxymethylsilane/nanoparticle ratio, suggesting the importance of alkyl chain length in forming a hydrophobic network. More importantly, by functionalising the nanoparticles with a mixture of triethoxymethylsilane and trimethoxy(octyl)silane and hence incorporating both functional groups onto the surface of the nanoparticles, it was shown that the homogeneity of the octyl-chain network was crucial for hydrophobicity, as increasing the triethoxymethylsilane to trimethoxy(octyl)silane ratio to 160:40 (v/v) could reduce the water contact angle significantly from $148.4^{\circ} \pm 3.5^{\circ}(60-200 \mu \mathrm{L}$ trimethoxy (octyl)silane $\cdot \mathrm{g}^{-1}$ ) to $30.5^{\circ} \pm 1.0^{\circ}$ ( $40 \mu \mathrm{L}$ trimethoxy(octyl) silane $\left.\cdot \mathrm{g}^{-1}\right)$. The findings in this study are useful for the intelligent design of surface with tuneable hydrophobicity in environmentally relevant applications.

Acknowledgements This study is part of the $\mathrm{SCoBiC}$ project funded by the UK's EPSRC (EP/N015916/1). The authors declare no conflict of interests.

Electronic Supplementary Material Supplementary material is available in the online version of this article at https://doi.org/10.1007/s11705-020$2003-0$ and is accessible for authorized users.

Open Access This article is licensed under a Creative Commons Attribution 4.0 International License, which permits use, sharing, adaptation, distribution and reproduction in any medium or format, as long as you give appropriate credit to the original author(s) and the source, provide a link to the Creative Commons licence, and indicate if changes were made. The images or other third party material in this article are included in the article's Creative Commons licence, unless indicated otherwise in a credit line to the material. If material is not included in the article's Creative Commons licence and your 
intended use is not permitted by statutory regulation or exceeds the permitted use, you will need to obtain permission directly from the copyright holder. To view a copy of this licence, visit http://creativecommons.org/licenses/by/4.0/.

\section{References}

1. Sun X, Zhang Y, Chen G, Gai Z. Application of nanoparticles in enhanced oil recovery: a critical review of recent progress. Energies, 2017, 10(3): 345

2. Behzadi A, Mohammadi A. Environmentally responsive surfacemodified silica nanoparticles for enhanced oil recovery. Journal of Nanoparticle Research, 2016, 18(9): 266

3. Rognmo A U, Heldal S, Fernø M A. Silica nanoparticles to stabilize $\mathrm{CO}_{2}$-foam for improved $\mathrm{CO}_{2}$ utilization: enhanced $\mathrm{CO}_{2}$ storage and oil recovery from mature oil reservoirs. Fuel, 2018, 216: 621-626

4. Yang X, Shen Z, Zhang B, Yang J, Hong W X, Zhuang Z, Liu J. Silica nanoparticles capture atmospheric lead: implications in the treatment of environmental heavy metal pollution. Chemosphere, 2013, 90(2): 653-656

5. Stöber W, Fink A, Bohn E. Controlled growth of monodisperse silica spheres in the micron size range. Journal of Colloid and Interface Science, 1968, 26(1): 62-69

6. Bogush G H, Tracy M A, Zukoski Iv C F. Preparation of monodisperse silica particles: control of size and mass fraction. Journal of Non-Crystalline Solids, 1988, 104(1): 95-106

7. Park S K, Kim K D, Kim H T. Preparation of silica nanoparticles: determination of the optimal synthesis conditions for small and uniform particles. Colloids and Surfaces. A, Physicochemical and Engineering Aspects, 2002, 197(1-3): 7-17

8. Costa C A, Leite C A, Galembeck F. Size dependence of Stöber silica nanoparticle microchemistry. Journal of Physical Chemistry B, 2003, 107(20): 4747-4755

9. Green D L, Lin J S, Lam Y F, Hu M C, Schaefer D W, Harris M T. Size, volume fraction, and nucleation of Stober silica nanoparticles. Journal of Colloid and Interface Science, 2003, 266(2): 346-358

10. Nozawa K, Gailhanou H, Raison L, Panizza P, Ushiki H, Sellier E, Delville J P, Delville M H. Smart control of monodisperse Stöber silica particles: effect of reactant addition rate on growth process. Langmuir, 2005, 21(4): 1516-1523

11. Masalov V M, Sukhinina N S, Kudrenko E A, Emelchenko G A. Mechanism of formation and nanostructure of Stöber silica particles. Nanotechnology, 2011, 22(27): 275718

12. Li S, Wan Q, Qin Z, Fu Y, Gu Y. Understanding Stöber silica's pore characteristics measured by gas adsorption. Langmuir, 2015, 31(2): 824-832

13. Greasley S L, Page S J, Sirovica S, Chen S, Martin R A, Riveiro A, Hanna J V, Porter A E, Jones J R. Controlling particle size in the Stöber process and incorporation of calcium. Journal of Colloid and Interface Science, 2016, 469: 213-223

14. Liberman A, Mendez N, Trogler W C, Kummel A C. Synthesis and surface functionalization of silica nanoparticles for nanomedicine. Surface Science Reports, 2014, 69(2-3): 132-158

15. Sawada H, Tashima T, Nishiyama Y, Kikuchi M, Goto Y, Kostov G, Ameduri B. Iodine transfer terpolymerization of vinylidene fluoride, $\alpha$-trifluoromethacrylic acid and hexafluoropropylene for exceptional thermostable fluoropolymers/silica nanocomposites. Macromolecules, 2011, 44(5): 1114-1124

16. Kobayashi M, Juillerat F, Galletto P, Bowen P, Borkovec M. Aggregation and charging of colloidal silica particles: effect of particle size. Langmuir, 2005, 21(13): 5761-5769

17. Binks B P, Lumsdon S O. Influence of particle wettability on the type and stability of surfactant-free emulsions. Langmuir, 2000, 16 (23): 8622-8631

18. Aveyard R, Binks B P, Clint J H. Emulsions stabilised solely by colloidal particles. Advances in Colloid and Interface Science, 2003, 100: 503-546

19. Balard H, Papirer E, Khalfi A, Barthel H. Trimethylchlorosilane modified silica surfaces: characterization by inverse gas chromatography using PDMS oligomers as probes. Composite Interfaces, 1998, 6(1): 19-25

20. Ghaleh V R, Mohammadi A. The stability and surface activity of environmentally responsive surface-modified silica nanoparticles: the importance of hydrophobicity. Journal of Dispersion Science and Technology, 2020, 41(9): 1299-1310

21. Zhao B, Zhu L. Mixed polymer brush-grafted particles: a new class of environmentally responsive nanostructured materials. Macromolecules, 2009, 42(24): 9369-9383

22. Wang Y, Fan D, He J, Yang Y. Silica nanoparticle covered with mixed polymer brushes as Janus particles at water/oil interface. Colloid \& Polymer Science, 2011, 289(17-18): 1885-1894

23. Pyun J, Jia S, Kowalewski T, Patterson G D, Matyjaszewski K. Synthesis and characterization of organic/inorganic hybrid nanoparticles: kinetics of surface-initiated atom transfer radical polymerization and morphology of hybrid nanoparticle ultrathin films. Macromolecules, 2003, 36(14): 5094-5104

24. Worthen A J, Tran V, Cornell K A, Truskett T M, Johnston K P. Steric stabilization of nanoparticles with grafted low molecular weight ligands in highly concentrated brines including divalent ions. Soft Matter, 2016, 12(7): 2025-2039

25. Schultz J A, Lavielle L, Martin C. The role of the interface in carbon fibre-epoxy composites. Journal of Adhesion, 1987, 23(1): 45-60

26. Della Volpe C, Siboni S. Some reflections on acid-base solid surface free energy theories. Journal of Colloid and Interface Science, 1997, 195(1): 121-136

27. Das S C, Larson I, Morton D A, Stewart P J. Determination of the polar and total surface energy distributions of particulates by inverse gas chromatography. Langmuir, 2011, 27(2): 521-523

28. Van Oss C J, Chaudhury M K, Good R J. Interfacial Lifshitz-van der Waals and polar interactions in macroscopic systems. Chemical Reviews, 1988, 88(6): 927-941

29. Heng J Y, Thielmann F, Williams D R. The effects of milling on the surface properties of form I paracetamol crystals. Pharmaceutical Research, 2006, 23(8): 1918-1927

30. Ho R, Heng J Y. A review of inverse gas chromatography and its development as a tool to characterize anisotropic surface properties of pharmaceutical solids. Kona Powder and Particle Journal, 2013, 30(0): 164-180

31. Karde V, Ghoroi C. Influence of surface modification on wettability and surface energy characteristics of pharmaceutical excipient powders. International Journal of Pharmaceutics, 2014, 475(1-2): $351-363$ 
32. Ramanaiah S, Karde V, Venkateswarlu P, Ghoroi C. Effect of temperature on the surface free energy and acid-base properties of Gabapentin and Pregabalin drugs - a comparative study. RSC Advances, 2015, 5(60): 48712-48719

33. Karde V, Ghoroi C. Fine powder flow under humid environmental conditions from the perspective of surface energy. International Journal of Pharmaceutics, 2015, 485(1-2): 192-201

34. Jafarzadeh M, Adnan R, Mazlan M K. Thermal stability and optical property of ormocers (organically modified ceramics) nanoparticles produced from copolymerization between amino-silanes and tetraethoxysilane. Journal of Non-Crystalline Solids, 2012, 358 (22): $2981-2987$
35. Wu F, Zhang B, Yang W, Liu Z, Yang M. Inorganic silica functionalized with PLLA chains via grafting methods to enhance the melt strength of PLLA/silica nanocomposites. Polymer, 2014, 55 (22): $5760-5772$

36. Sándor M, Nistor C, Szalontai G, Stoica R, Nicolae C, Alexandrescu E, Fazakas J, Oancea F, Donescu D. Aminopropyl-silica hybrid particles as supports for humic acids immobilization. Materials (Basel), 2016, 9(1): 34

37. Yuan W, Wang F, Chen Z, Gao C, Liu P, Ding Y, Zhang S, Yang M. Efficient grafting of polypropylene onto silica nanoparticles and the properties of PP/PP-g-SiO 2 nanocomposites. Polymer, 2018, 151: $242-249$ 